${ }^{1}$ State University of Feira de Santana, Department of Health, Feira de Santana, Bahia, Brazil.

${ }^{2}$ São Judas Tadeu University, Department of Operative Dentistry, Sao Paulo, Sao Paulo, Brazil.

${ }^{3}$ State University of Feira de Santana, Department of Biological Sciences, Feira de Santana, Bahia, Brazil.
* Corresponding author: Ynara Bosco de Oliveira Lima-Arsati UEFS - Av. Transnordestina, s/n. Bairro Novo Horizonte, Feira de Santana, Bahia, Brasil. ZIP CODE: 44036-900. Phone/Fax: (+55) 75 3161-8019.

Email: ynaralima76@gmail.com

Received: August 22, 2020

Accepted: December 14, 2020

\section{In vitro determination of potentially bioavailable fluoride in diet and toothpaste after ingestion}

\author{
Cristiane Brandão Santos Almeida ${ }^{1}$ (iD), José Augusto \\ Rodrigues $^{2}$ (ID), Valéria Souza Freitas ${ }^{1}$ (iD, Ynara \\ Bosco de Oliveira Lima-Arsati ${ }^{3, *}$ (iD
}

Aim: To propose a new method to determine in vitro potentially bioavailable fluoride ( $F$ ) in diet and toothpaste after ingestion. Methods: Diet samples (D) were obtained from 15 portions of a meal served to children in a day care centre. To simulate the ingestion of toothpaste during brushing after meals, a specific amount of toothpaste was added to the diet samples $(D+T)$. $F$ was determined in $D$ and $D+T$ after incubation in a solution that simulated "gastric juice" (0.01 M hydrochloric acid) at $37^{\circ} \mathrm{C}$ for 30, 60 and 120 min. Microdiffusion facilitated by HMDS was used to determine the total $F$ concentrations in samples $D$ and $D+T$. The analyses were performed using an ion specific electrode. Results: For D samples, incubation in "gastric juice" for 30, 60 and 120 min resulted in $F$ concentrations ( $\mu \mathrm{g} \mathrm{F/mL)}$ of $0.75 \pm 0.06^{\mathrm{c}}, 0.77 \pm 0.07^{\mathrm{c}}$ and $0.91 \pm 0.09^{\mathrm{b}}$, corresponding to $75.3,77.3$ and $90.7 \%$ of the total $F\left(1.02 \pm 0.12^{a}\right)$, respectively $(p=0.0001$; ANOVA + Tukey). For D + T samples, these values of $F$ concentrations $(\mu \mathrm{g} \mathrm{F} / \mathrm{mL})$ were $2.55 \pm 0.46^{\mathrm{b}}, 2.83 \pm 0.44^{\mathrm{ab}}$ and $3.15 \pm 0.37$, corresponding to $86.9,94.8$ and $106.7 \%$ of the total $F\left(2.99 \pm 0.34^{a}\right)$, respectively $(p=0.0023$; ANOVA + Tukey). Conclusion: Then, it can be concluded that the proposed method of "gastric juice" is a promising protocol for determining potentially bioavailable fluoride in the diet and toothpaste after ingestion. However, additional studies are desirable.

Keywords: Fluoride. Dentifrice. Toothpaste. Diet. Dental Fluorosis. 


\section{Introduction}

Several studies were developed to estimate the effect of fluoride $(F)$ intake and risk of dental fluorosis development. The mean intake of $\mathrm{F}$ from diet and toothpaste observed in scientific studies ranged from 0.036 to $0.090 \mathrm{mg} \mathrm{F} / \mathrm{kg} /$ day ${ }^{1-7}$. However, the correlation between high doses of $\mathrm{F}$ exposure and high prevalence of dental fluorosis has not been established yet ${ }^{8-9}$. This is probably because the doses determined may be overestimated for two reasons: the daily brushing frequency is not as high as that reported ${ }^{3}$ and the fact that the dose is based on the amount of ingested $F$, rather than absorbed.

In vivo studies demonstrate that the presence of food in the stomach decreases the absorption of $\mathrm{F}$ from the toothpaste ingested during brushing after regular meals ${ }^{10}$. The reason is probably the increase in the $\mathrm{pH}$ of the stomach or the formation of low solubility salts between $\mathrm{F}$ and cations calcium $\left(\mathrm{Ca}^{2+}\right)$, aluminum $\left(\mathrm{Al}^{3+}\right)$ and magnesium $\left(\mathrm{Mg}^{2+}\right)$ from food. Another aspect to consider is the type of abrasive present in the toothpaste. Toothpastes with calcium-based abrasives, such as calcium carbonate $\left(\mathrm{CaCO}_{3}\right)$ have part of the insoluble $\mathrm{F}$, because it is bounded to calcium. The silica-based abrasive toothpastes present the entire $F$ in the soluble form, being bioavailable for absorption ${ }^{11}$.

Thus, the reported dose values may be overestimated, as discussed in some of these studies $^{3,8}$. When $\mathrm{F}$ is needed to be quantified in food samples using ion-specific electrode, it is necessary to extract the $F$ ion $\left(F^{-}\right)$, since the electrode can only detect $F$ ion in solution ${ }^{12-13}$. The recommended method to determine $F$ in food samples is microdiffusion facilitated by HMDS ${ }^{14-15}$, originally described by Taves ${ }^{14}$ (1968), and manages to extract total $F$ of the samples, even solid ones, besides concentrating them approximately 6.7 times, which increases the sensitivity of the method. However, it is a laborious, expensive, and time-consuming technique.

Considering the use of different protocols for microdiffusion, Martínez-Mier et al. ${ }^{15}$ (2011) demonstrated that a standardization of techniques increased the recovery of $\mathrm{F}$ and resulted in very precise and exact values between different laboratories. For food samples, they advocated the use of microdiffusion in a protocol which has some differences from the original technique, in addition to having the same critical step of completing the final volume of the drops with deionized water to compensate for evaporation.

Although the protocols for total $\mathrm{F}$ extraction are established, not all $\mathrm{F}$ is soluble in food and cannot be absorbed. Therefore, methods that can determine potentially bioavailable fluoride are desirable to improve the discussion about $\mathrm{F}$ intake and the risk of dental fluorosis ${ }^{16}$. It was reported that the use of $0.01 \mathrm{M} \mathrm{HCl}$ to dissolve samples of prenatal supplements resulted in the recovery of $38.3 \%$ of the total $\mathrm{F}^{17}$. The authors suggested that body temperature and peristaltic movements should be simulated to provide more realistic results for potentially bioavailable $\mathrm{F}$ during digestion.

Considering that the main sources of systemic $\mathrm{F}$ for children at risk for dental fluorosis are diet (food and beverages) and toothpaste ${ }^{1-7}$, the aim of the present study was to propose a new method to determine in vitro the concentration of potentially bioavailable $\mathrm{F}$ in samples of diet and toothpaste. 


\section{Methodology}

\section{Experimental design}

This was an in vitro study. The experimental units were 15 diet samples, which were analyzed pure (D) or with toothpaste $(D+T)$; there were four experimental groups, corresponding to the method used to extract fluoride from the samples: "gastric juice" 30 min, "gastric juice" 60 min, "gastric juice" 120 min and microdiffusion facilitated by HMDS. The response variable was the F concentration, expressed in $\mu \mathrm{g} / \mathrm{mL}$.

\section{Obtaining diet samples (D)}

The sample size was statistically determined using the Bioestat software, based on data from previous studies, as follows: minimum difference between treatments $=0.15$; standard deviation $=0.1$; study power $=0.8$; level of significance $=0.05$. The result was 11 , but we used 15 just in case. After authorization from the Municipal Education Secretary of Feira de Santana, Bahia, diet samples were collected at a daycare center. The meals were prepared with optimally fluoridated water $(0.76 \pm 0.01 \mu \mathrm{g} \mathrm{F} / \mathrm{mL})$ and consisted of rice, beans, pasta and meat. The cook placed the meals on dishes as usual, reproducing the amount usually consumed by children aged 2 to 3 years during lunch. Fifteen dishes were selected at random and each meal was collected separately in plastic containers. In the laboratory, samples of the diet were weighed, $100 \mathrm{~mL}$ of distilled and deionized water were added to each sample and homogenized with a mixer, without a filtration method. The final volume was determined, and each sample was frozen $\left(-18^{\circ} \mathrm{C}\right)$ until analysis. Knowing its volume before and after adding water, the dilution factor was calculated for each one.

\section{Obtaining diet + toothpaste samples $(\mathrm{D}+\mathrm{T})$}

The used toothpaste was Tandy® (Colgate, strawberry flavor, lot 6289BR121K, validity 10/19), containing $942.8 \pm 3.8 \mu \mathrm{g} \mathrm{F} / \mathrm{g}$ as total soluble fluoride (TSF), from sodium fluoride. The TSF concentration was determined by the direct method using an ion-specific electrode ${ }^{18}$.

According to a previous study ${ }^{7}$, children residing in Feira de Santana, Bahia, aged between 15 and 30 months, used an average of $0.47 \mathrm{~g}$ of toothpaste for brushing their teeth, and ingested $70.5 \%$ of this, resulting in $0.33 \mathrm{~g}$ of toothpaste ingested. In the present study, the average volume of the diet collected at the daycare center, simulating lunch, was $250 \mathrm{~mL}$. Hypothetically considering that children would brush their teeth right after lunch, the gastric content of these children would be $250 \mathrm{~mL}$ of diet and $0.33 \mathrm{~g}$ of toothpaste. We used this proportion to determine the amount of toothpaste to be added to the diet + toothpaste samples $(D+T)$, considering the volume of diet used in each analytic method: $\mathrm{Ta}=\mathrm{Da}$ * Ti/ Di

Where:

- Ta: weight of toothpaste to be added to sample in laboratory analysis (g)

- Da: volume of diet used in laboratory analysis $(\mathrm{mL})$

- Ti: estimated weight of toothpaste ingested during toothbrushing (g)

- Di: estimated volume of diet ingested per meal $(\mathrm{mL})$ 
So, for microdiffusion facilitated by HMDS, Ta $=3 * 0.33 / 250=0.004 \mathrm{~g}$. And for the "gastric juice", $\mathrm{Ta}=7$ *0.33/ $250=0.009 \mathrm{~g}$.

\section{F determination using the "gastric juice" method (shown in Fig. 1)}

This simulation was performed by incubation of the samples in a solution that simulated gastric juice ( $0.01 \mathrm{M}$ hydrochloric acid) at $37^{\circ} \mathrm{C}$ for 30,60 and $120 \mathrm{~min}$. This solution was called "gastric juice". This protocol was based on the reported by Fernandes and Cury" (1993), and in a preliminary study of our group ${ }^{19}$, which showed that the 7:1 proportion of sample to "gastric juice" resulted in a pH value corresponding to the gastric content during digestion ( $\mathrm{pH}=4.76^{12}$ ). In addition, the preliminary study showed that the incubation in "gastric juice" for 120 min resulted in a recovery of $95.51 \%$ of total $F$ concentrations in samples. So, we decided to test this incubation time (120 min) against lower times (60 and $30 \mathrm{~min}$ ).

D samples were prepared adding $1 \mathrm{~mL}$ of $0.01 \mathrm{M} \mathrm{HCl}$ to $7 \mathrm{~mL}$ of diet. D+T samples were prepared adding $1 \mathrm{~mL}$ of $0.01 \mathrm{M} \mathrm{HCl}$ to $7 \mathrm{~mL}$ of diet and $0.009 \mathrm{~g}$ of toothpaste (based on the formulae previously demonstrated: $\mathrm{Ta}=\mathrm{Da} * \mathrm{Ti} / \mathrm{Di}$ ). Plastic tubes containing the samples were incubated for 30, 60 or 120 min in an oven (Sterilifer, SX 300) at $37^{\circ} \mathrm{C}$. Then centrifugation (Thermo Scientific) was performed for $5 \mathrm{~min}$ at 10,000 rpm. Fat was cautiously removed from the surface using an absorbent paper, and $0.4 \mathrm{~mL}$ of supernatant was buffered with $0.4 \mathrm{~mL}$ of TISAB II for $F$ analysis.

The F concentration was determined by means of an ion-specific electrode (ISE; Orion Model 96-09, Orion Research Incorporated, Cambridge, MA, USA) and an ion analyzer (Orion Star A214, Orion Research Incorporated), previously calibrated with standards solutions $(0.4,0.8,1.6,3.2$ and $6.4 \mu \mathrm{g} / \mathrm{mL})$ in triplicate, prepared in the same conditions of the samples. The calibration and concentrations determined were tested in the linear regression curve, using Excel software, where a calculation program transformed the values of $\mathrm{mV}$ provided by the electrode in $\mathrm{F}$ concentration. Blank correction was done. $\mathrm{D}$ and $\mathrm{D}+\mathrm{T}$ samples were analyzed in separate days. For $\mathrm{D}$ samples, the mean variation between obtained and expected values for calibration curve was $0.0085 \%$ and R2 $=0.9998$. For D+T samples, the mean variation for calibration curve was $0.001 \%$ and R2 $=0.9999$.

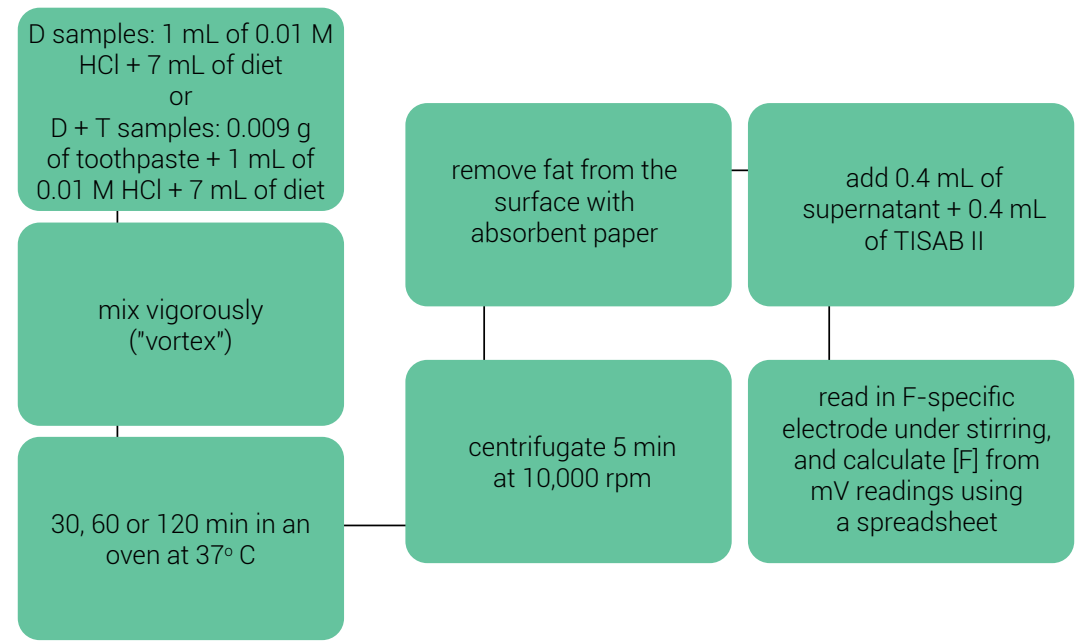

Figure 1. F determination using the "gastric juice" method. 


\section{F determination using microdiffusion facilitated by HMDS (shown in Fig. 2)}

It was based on the method described by Taves ${ }^{14}$ (1968). A cap from a plastic tube was placed, using vaseline, in the center of a plastic petri dish. For D samples, $3 \mathrm{~mL}$ of homogenized diet was added to the petri dish. For D+T samples, before the diet, $0.004 \mathrm{~g}$ of toothpaste was added to the petri dish (based on the formulae previously demonstrated: $\mathrm{Ta}=\mathrm{Da}$ * Ti/ Di). For calibration curve, $1 \mathrm{~mL}$ of standard solution and $2 \mathrm{~mL}$ of distilled and deionized water were added to the petri dish. Then, $0.10 \mathrm{~mL}$ of $1.65 \mathrm{M}$ sodium hydroxide was placed in the cap. The petri dish was closed, sealed with vaseline and $1.0 \mathrm{~mL}$ of $6 \mathrm{M}$ hydrochloric acid saturated with HMDS added to the sample through a hole made in the petri dish cap. The hole was sealed with vaseline and the petri dish was shaken at room temperature for $14 \mathrm{~h}$ in an orbital shaker (Kline, NT-150). Then, the plastic cap containing $F$ diffused from the sample was dried at $60^{\circ} \mathrm{C}$ for $2 \mathrm{~h}$ in an oven (Sterilifer, SX 300). This cap was then used to close a plastic tube containing $0.40 \mathrm{~mL}$ of $0.66 \mathrm{M}$ acetic acid. The tube was inverted and vigorously shaken to dissolve the fluoride crystals present in the cap.

The $\mathrm{F}$ concentration was determined by means of ISE, previously calibrated with standards solutions $(0.4,0.8,1.6,3.2$ and $6.4 \mu \mathrm{g} \mathrm{F} / \mathrm{mL})$ in triplicate, prepared in the same conditions of the samples. Blank correction was done. D and D+T samples were analyzed in separate days. For $\mathrm{D}$ samples, the mean variation between obtained and expected values for calibration curve was $0.054 \%$ and R2 $=0.9989$. For D+T samples, the mean variation for calibration curve was $1.423 \%$ and R2 $=0.9527$.

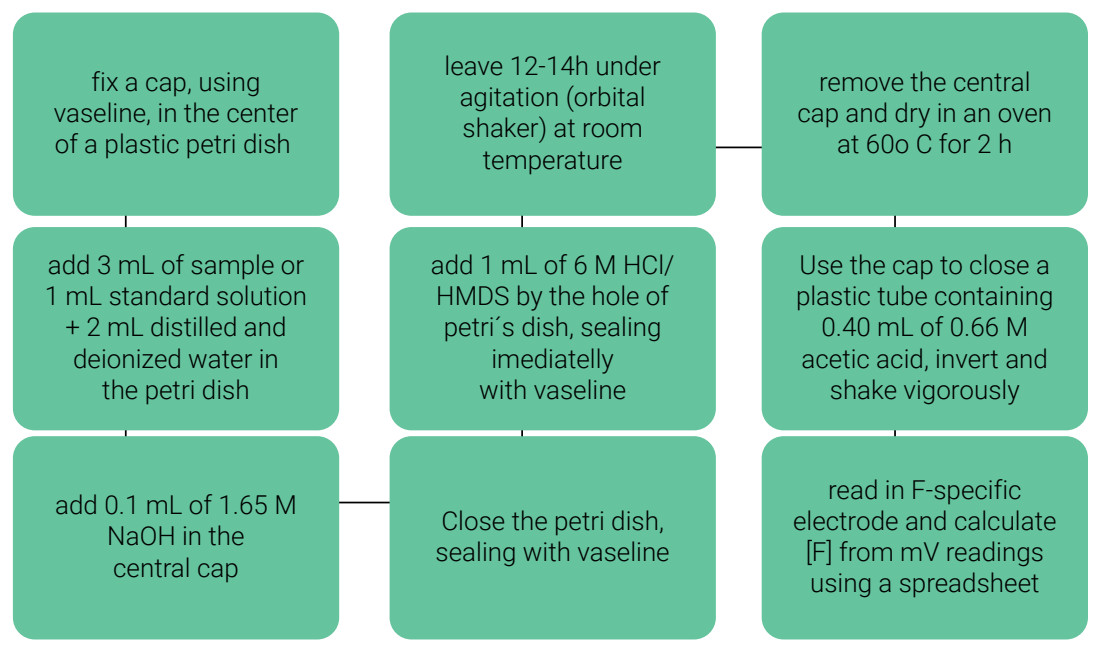

Figure 2. F determination using microdiffusion facilitated by HMDS.

\section{Statistical analysis}

A descriptive statistical analysis was performed to obtain values of central tendency and dispersion. Then, Analysis of Variance was used to compare the results of $F$ concentration obtained for the different methods (one-way ANOVA + Tukey test). The coefficient of variation (CV\%) and the intraclass correlation coefficient (ICC) were determined for all samples, analyzed in triplicate, to evaluate reproducibility. The software BioEstat 5.0 and SPSS were used; the level of significance was 5\%. 


\section{Results}

Results are expressed in table 1. Regarding diet (D) samples, "gastric juice" resulted in lower $\mathrm{F}$ concentrations than the microdiffusion facilitated by HMDS. Comparison among incubation times in "gastric juice" showed that 120 min resulted in higher $F$ concentration than 30 and 60 min. Both the "gastric juice" method (CV\% $=4.79 \%$ and ICC $=0.86)$ and the HMDS-facilitated microdiffusion (CV\% $=6.58 \%$ and ICC $=0.89$ ) showed high reproducibility ${ }^{20}$.

The diet + toothpaste $(D+T)$ samples comparison showed that the "gastric juice" with 60 and 120 min of incubation did not differ from HMDS-facilitated microdiffusion. The "gastric juice" method (CV\% $=6.68 \%$ and ICC $=0.88)$ showed high reproducibility, but the HMDS-facilitated microdiffusion (CV\% $=10.45 \%$ and $I C C=0.42$ ) showed low reproducibility.

Table 1. Mean, standard deviation and range of $F$ concentration ( $\mu \mathrm{g} F / \mathrm{mL}$ ) in diet samples (D) and diet+toothpaste samples $(D+T)$, using different methods of $F$ extraction ${ }^{\text {. }}$.

\begin{tabular}{|c|c|c|c|}
\hline \multicolumn{2}{|l|}{ Method } & $\begin{array}{l}\text { Diet (D) } \\
(n=15)\end{array}$ & $\begin{array}{l}\text { Diet+Toothpaste }(D+T) ; \\
(n=15)\end{array}$ \\
\hline \multicolumn{2}{|c|}{ microdiffusion facilitated by HMDS } & $\begin{array}{c}1.02 \pm 0.12 a \\
(0.77-1.25)\end{array}$ & $\begin{array}{c}2.99 \pm 0.34 \mathrm{a} \\
(2.70-4.06)\end{array}$ \\
\hline \multirow{3}{*}{ "gastric juice" } & $30 \min$ & $\begin{array}{c}0.75 \pm 0.06 c \\
(0.63-0.88)\end{array}$ & $\begin{array}{l}2.55 \pm 0.46 b \\
(1.25-3.16)\end{array}$ \\
\hline & $60 \min$ & $\begin{array}{c}0.77 \pm 0.07 c \\
(0.69-0.92)\end{array}$ & $\begin{array}{c}2.83 \pm 0.44 \mathrm{ab} \\
(2.24-3.85)\end{array}$ \\
\hline & $120 \mathrm{~min}$ & $\begin{array}{c}0.91 \pm 0.09 b \\
(0.78-1.09)\end{array}$ & $\begin{array}{c}3.15 \pm 0.37 a \\
(2.49-3.96)\end{array}$ \\
\hline
\end{tabular}

a Values followed by distinct letters indicate a statistically significant difference between the analytical techniques within each group ( $D$ or $D+T)(P<0.05$; One-way ANOVA and Tukey's test).

Considering the results of the microdiffusion method as total $F$ concentration, it was found that, using "gastric juice" for $30 \mathrm{~min}, 75.25 \%( \pm 10.77)$ of the total F were extracted from the samples of the diet. These values for 60 and 120 min were $77.33 \%$ $( \pm 12.80)$ and $90.70 \%( \pm 9.69)$, respectively. For the samples of diet + toothpaste, using "gastric juice" for 30, 60 and 120 min extracted $86.90 \%$ ( \pm 18.39$), 94.83 \%( \pm 10.06)$ and $106.65 \%( \pm 15.96)$ of total $F$, respectively.

\section{Discussion}

After ingestion, the potentially bioavailable fluoride is represented by its soluble and in ionic form $(\mathrm{F}-$ ), which can be converted into hydrofluoric acid (HF) and absorbed, having some systemic effect. The formation of HF depends on the $\mathrm{pH}$ of the medium. Gastric $\mathrm{pH}$ is acidic due to the presence of hydrochloric acid $(\mathrm{HCl})$ in gastric juice. In addition to $\mathrm{HCl}$, gastric juice is composed of pepsinogen, intrinsic factor and mucus. The rate of $\mathrm{HCl}$ secretion varies depending on the stimuli. When the stomach is "empty", it contains about $50 \mathrm{~mL}$ of gastric juice and its $\mathrm{pH}$ is approximately $2^{21}$. After eating, the $\mathrm{pH}$ increases to $4.1-6.3^{22}$. Therefore, it is necessary to assess the presence of potentially bioavailable fluoride in these conditions.

In laboratory analysis, the electrode can detect $\mathrm{F}^{-}$. TISAB II is generally used as a buffer, in order to regulate the $\mathrm{pH}$ about 5.5 , in which most of the soluble $\mathrm{F}$ will be 
in ionic form $(\mathrm{F})^{23}$. It must be considered that not all the $\mathrm{F}$ detected by the electrode represents the absorbed $F$, as there are different conditions for it to be detected in the laboratory (to be in the $\mathrm{F}^{-}$form) ${ }^{23}$ and to be absorbed in vivo (to be in the HF form) ${ }^{24}$.

In an in vivo situation, perhaps the main reason for the difference between ingested and absorbed $\mathrm{F}$ is related to gastric $\mathrm{pH}$. The $\mathrm{F}$ will only be absorbed in the form of hydrofluoric acid $(\mathrm{HF})^{24}$, which is predominant when the $\mathrm{pH}$ of the gastrointestinal tract is less than 3.2 (pka value of HF). Therefore, depending on the gastric content, the $\mathrm{pH}$ might be different and therefore the absorption of $\mathrm{F}$ might also be different. Considering the gastric $\mathrm{pH}$ between 4.1 and 6.3, the predominant $\mathrm{F}$ will be in the ionic form $(F)$, therefore, it will not be absorbed ${ }^{24}$. The "gastric juice" method was proposed to simulate gastric conditions during digestion ( $\mathrm{pH}$ and temperature). Thus, when the samples were subjected to these conditions, it was assumed that $F$ would be solubilized in the same way that it occurs in real conditions.

The results showed that there was no difference in $F$ concentration obtained by using microdiffusion ou "gastric juice" for 120 min to extract F from samples of diet. It means that "gastric juice" method, with 120 min of incubation, can extract total $F$ from diet samples. This is an important result, considering the advantages of the "gastric juice" method (simplicity and cost). The amount of F detected by the electrode analysis represents the ionic $F$, which might not be totally absorbed, since not all $F^{-}$will form HF. Therefore, despite being potentially bioavailable, most of the $F$ in the diet will not be absorbed. This could also justify the lack of correlation between $F$ intake and dental fluorosis reported in the literature ${ }^{8-9}$.

However, when the diet samples were incubated in "gastric juice" for shorter periods, less potentially bioavailable $F$ was detected, showing that in the "gastric juice" method, the time of incubation affected the extraction of $F$ from the diet samples. In fact, this method actually extracts fluoride from the samples, instead of just detecting the one already soluble; it is only a matter of time. Then additional studies are needed, to correlate shorter and longer incubation periods to in vivo conditions and assess the relationship between incubation time and potentially bioavailable $F$ extraction from diet samples.

Considering the samples of diet + toothpaste, it is possible that the duration of the incubation was important only for $F$ in the diet, since the $F$ concentrations were lower than the total $F$ concentration only for the group incubated for 30 min. Perhaps the $F$ in toothpaste, which contained sodium fluoride ( $\mathrm{NaF}$ ) and was already in ionic form, remained so, without being inactivated by cations present in food.

It is also necessary to note that $D+T$ analyzes using microdiffusion showed low reproducibility (ICC $=0.42$ ). This was not expected, since microdiffusion is a well-established methodology for food ${ }^{15}$, and the toothpaste used contained sodium fluoride. Our hypothesis is that the toothpaste samples dried out because they were the first to be added (weighed). Only after toothpaste was added to all Petri dishes, the diet (homogenized food) was added. Although the Petri dish remained under agitation (orbital shaker) overnight, the toothpaste may not have adequately solubilized in food. To avoid this, it is suggested to add toothpaste after the diet.

Among the limitations of the "gastric juice" method, is the fact that it is not as dynamic as the digestion process, where $\mathrm{HCl}$ secretions are not constant, so neither is the 
$\mathrm{pH}$. In addition, gastric juice is not exclusively composed of $\mathrm{HCl}$. Another limitation was that the samples were not under agitation while incubated at $37^{\circ} \mathrm{C}$, therefore, peristaltic movements were not simulated, as suggested by Fernandes and Cury ${ }^{17}$ (1993). For the "gastric juice" method to be considered adequate to determine potentially bioavailable F, additional studies must be conducted, evaluating the effect of the following factors: absence of incubation time; repetition of analyzes over time (short periods) in the same sample until [F] reaches a plateau; addition and recovery of known amounts of $F$ to samples. In addition, it must be considered that not all potentially bioavailable $F$ will be absorbed, the "gastric juice" technique would also overestimate the dose of systemic exposure to fluoride and, consequently, the risk of dental fluorosis. Therefore, clinical studies, using validated biological samples that reflect fluoride metabolism are essential to complement in vitro studies ${ }^{16}$.

Then, it can be concluded that the proposed method of "gastric juice" is a promising protocol for determining potentially bioavailable fluoride in the diet and toothpaste after ingestion. However, additional studies are desirable.

\section{Acknowledgements}

We are grateful to FAPESB (Bahia Research Foundation) for the master's degree granting (Process n. BOL 93/2016) and financial support (FAPESB/CNPq, n. 485/2011).

\section{References}

1. Guha-Chowdhury N, Drummond BK, Smillie AC. Total fluoride intake in children aged 3 to 4 years - a longitudinal study. J Dent Res. 1996 Jul;75(7):1451-7. doi: 10.1177/00220345960750070401.

2. Rojas-Sanchez F, Kelly SA, Drake KM, Eckert GJ, Stookey GK, Dunipace AJ. Fluoride intake from foods, beverages and dentifrice by young children in communities with negligibly and optimally fluoridated water: a pilot study. Community Dent Oral Epidemiol. 1999 Aug;27(4):288-97. doi: 10.1111/j.1600-0528.1998.tb02023.x.

3. Lima YBO, Cury JA. [Fluoride intake by children from water and dentifrice]. Rev Saude Publica. 2001;35(6):576-81. doi: 10.1590/S0034-89102001000600012. Portuguese.

4. Paiva SM, Lima YBO, Cury JA. Fluoride intake by Brazilian children from two communities with fluoridated water. Comm Dent Oral Epidemiol. 2003 Jun;31(3):184-91. doi: 10.1034/j.16000528.2003.00035.x.

5. Omena LMF, Silva MF, Pinheiro CC, Cavalcante JC, Sampaio FC. Fluoride intake from drinking water and dentifrice by children living in a tropical area of Brazil. J Appl Oral Sci. 2006 Oct;14(5):382-7. doi: 10.1590/s1678-77572006000500015.

6. Lima CV, Cury JA, Vale GC, Lima MD, Moura LF, Moura MS. Total fluoride intake by children frmo a tropical Brazilian city. Caries Res. 2015;49(6):640-6. doi: 10.1159/000442029.

7. Lima-Arsati YBO, Gomes ARLF, Santos HKA, Arsati F, Oliveira MC, Freitas VS. Exposure to fluoride of children during the critical age for dental fluorosis, in the semiarid region of Brazil. Cien Saude Colet. 2018 Apr;23(4):1045-1054. doi: 10.1590/1413-81232018234.07952016. Portuguese.

8. Martins CC, Paiva SM, Lima-Arsati YBO, Ramos-Jorge ML, Cury JA. Prospective study of the association between fluoride intake and dental fluorosis in permanent teeth. Caries Res. 2008;42(2):125-33. doi: 10.1159/000119520. 
9. Warren JJ, Levy SM, Broffitt B, Cavanaugh JE, Kanellis MJ, Weber-Gasparoni K. Considerations on optimal fluoride intake using dental fluorosis and dental caries outcomes - a longitudinal study. J Public Health Dent. 2009;69(2):111-5. doi: 10.1111/j.1752-7325.2008.00108.x.

10. Cury JA, Del Fiol FS, Tenuta LMA. Low-fluoride dentifrice and gastrointestinal fluoride absorption after meals. J Dent Res. 2005 Dec;84(12):1133-7. doi: 10.1177/154405910508401208.

11. Falcão A, Tenuta LMA, Cury JA. Fluoride gastrointestinal absorption from Na2FPO3 /CaCO3 - and NaF/SiO2 - based toothpastes. Caries Res. 2013;47(3):226-33. doi: 10.1159/000346006.

12. Martinez-Mier EA, Tenuta LMA, Carey CM, Cury JA, Van Loveren C, Ekstrand KR, et al. ORCA Fluoride in Toothpaste Analysis Work Group. European Organization for Caries Research Workshop: Methodology for Determination of Potentially Available Fluoride in Toothpastes. Caries Res. 2019;53(2):119-36. doi: 10.1159/000490196.

13. Reshetnyak VY, Nesterova OV, Admakin OI, Dobrokhotov DA, Avertseva IN, Dostdar SA, et al Evaluation of free and total fluoride concentration in mouthwashes via measurement with ionselective electrode. BMC Oral Health. 2019 Nov 20;19(1):251. doi: 10.1186/s12903-019-0908-0.

14. Taves DR. Separation of fluoride by rapid diffusion using hexamethyldisiloxane. Talanta. 1968 Sep;15(9):969-74. doi: 10.1016/0039-9140(68)80097-9.

15. Martínez-Mier EA, Cury JA, Heilman JR, Katz BP, Levy SM, Li Y, et al. Development of gold standard ion-selective electrode-based methods for fluoride analysis. Caries Res. 2011;45(1):3-12. doi: $10.1159 / 000321657$.

16. Idowu OS, Azevedo LB, Valentine RA, Swan J, Vasantavada PV, Maguire A, et al. The use of urinary fluoride excretion to facilitate monitoring fluoride intake: a systematic scoping review. PLoS One. 2019 Sep;14(9):e0222260. doi: 10.1371/journal.pone.0222260.

17. Fernandes LMAG, Cury JA. [Prenatal fluoride - metabolic evaluation]. RBM Rev Bras Med. 1993 Nov;50(11):1546-52. Portuguese.

18. Cury JA, Oliveira MJL, Martins CC, Tenuta LM, Paiva SM. Available fluoride in toothpastes used by Brazilian children. Braz. Dent. J. 2010;21(5):396-400. doi: 10.1590/s0103-64402010000500003.

19. Lima-Arsati YBO, Santos HKA, Gomes ARLF. Evaluation of a protocol to simulate in vitro the decreased absorption of fluoride from toothpaste due to gastric content. In: XIX ABOPREV Meeting. Braz J Oral Sci. 2015;15(1):87. doi: 10.20396/bjos.v14i1.8641314.

20. Munro BH. Statistical methods for health care research. $3^{\text {rd }}$ ed. New York: Lippincott Williams \& Wilkins; 1997.

21. Hall JE. Guyton and Hall textbook of medical physiology. 13. ed. Philadelphia, PA: Elsevier; 2016.

22. McLauchlan G, Fullarton GM, Crean GP, McColl KE. Comparison of gastric body and antral pH: a 24 hour ambulatory study in healthy volunteers. Gut. 1989 May;30(5):573-8. doi: 10.1136/ gut.30.5.573.

23. Thermo Fisher Scientific. Thermo Scientific Orion Fluoride Ion Selective Electrode User Guide. 254792-001, Revision B. 2016 Sep [cited 2020 Jul 23]. Available from:

https://www.thermofisher.com/document-connect/document-connect. html?url=https\%3A\%2F\%2Fassets.thermofisher.com\%2FTFS-Assets\%2FLSG\%2Fmanuals\% 2FD15872 .pdf\&title=Rmx1b3JpZGUgSW9uIFNIbGVjdGI2ZSBFbGVjdHJVZGUgLSBVc2VylEd1aWRI.

24. Whitford GM. Absorption and plasma concentrations of fluoride. In: Whitford GM. The metabolism and toxicity of fluoride. 2. ed. Basel: Karger; 1996. Chapter 2, p.10-29. doi: 10.1159/ isbn.978-3-318-04022-7. 